\title{
Through-wall detection of human being with compressed UWB radar data
}

\author{
Baoju Zhang ${ }^{*}$ and Wei Wang
}

\begin{abstract}
The technology for through-wall human detection with ultra-wideband (UWB) radar was discussed. Due to the large amount of UWB radar data, compressive sensing theory was introduced and compressed UWB radar data can be collected. The singular value decomposition algorithm was used to acquire the singular values of compressed radar data. The compressed UWB radar data were collected at two statuses of human being for gypsum wall. The experimental results showed that the singular values with a human target were increased compared with those without a target.
\end{abstract}

Keywords: Through-wall human detection; UWB radar; Compressive sensing; Singular value decomposition

\section{Introduction}

Through-wall human detection is a new developing technology in recent years. It can penetrate non-metallic media (such as brick wall, ruins) to detect life signal in faroff areas but does not require any contact with the living body through electrodes or sensors [1-5]. Through-wall human detection is of great interest for many applications, such as military reconnaissance, anti-terrorism, and medical and natural disaster ambulance. Also, it has a positive significance in economic construction and social stability. Body electrostatic field, ultra-low-frequency electromagnetic energy, and ultra-wideband (UWB) radar technologies have been used for through-wall human detection currently.

The UWB radar can launch pulses of very short duration to penetrate non-metallic walls and obstacles. Due to its strong anti-interference ability, high-resolution performance, and good target recognition capabilities, the UWB radar has emerged as one of the most optimal choices for through-wall human detection. In [6], the detection of trapped human beings below rubble by means of M-sequence UWB radar was demonstrated, and a detection of buried people in wet rubble became possible by enhancing specific signal features using filtering algorithm. A further study of the above method has been proposed in [7-9]. The process of UWB radar signal has been presented which consists of the solution of the signal processing tasks such as raw radar data preprocessing, background subtraction, target detection, target trace estimation, target localization, and target tracking, and multiple target detection was also investigated. Also, for through-wall moving target, tracking by $\mathrm{M}$-sequence UWB radar was also described in [9] as a complex procedure consisting of such phases as raw radar data pre-processing, background subtraction, detection, time of arrival estimation, wall effect compensation, localization, and tracking itself. In [10], an ultra-wideband pseudo-noise radar was used for the detection of moving people and trapped people. The experimental results showed that the performance of antennas and of the radar electronics connected with powerful and clever algorithms were important for the successful application of human detection with UWB radar. In [11] and [12], some algorithms for through-wall human detection for three types of walls, that is gypsum wall, wooden door and brick wall, were discussed.

Although UWB radar technology can obtain good achievement in through-wall human detection, UWB radar generate large amounts of data and bring great pressure to data acquisition hardware. On the other hand, the data needed by the human being detection are far less than the actual sampling amount of data, which

\footnotetext{
* Correspondence: wdxyzbj@163.com

College of Physical and Electronic Information, Tianjin Normal University, Tianjin 300387, China
}

\section{黑 Springer}

(c) 2013 Zhang and Wang; licensee Springer. This is an Open Access article distributed under the terms of the Creative Commons Attribution License (http://creativecommons.org/licenses/by/2.0), which permits unrestricted use, distribution, and reproduction in any medium, provided the original work is properly cited. 
means that a lot of data collected are useless. In 2006, Donoho and Candès proposed a new theory called compressive sensing (or compressed sampling (CS)) based on signal decomposition and approximation theory $[13,14]$. The theory suggested that a high-dimensional signal can be projected into a low-dimensional space with a random measurement matrix when the signal was sparse or compressible. Then the original signal can be reconstructed from the low-dimensional information by solving an optimization problem. That is, the lowdimensional signal contained the main features of the original signal.

In this paper, we would propose an algorithm for through-wall human detection based on randomly collected UWB radar data with compressive sensing theory. The remainder of the paper is organized as follows: In Section 2, the compressive sensing theory will be introduced. Then the collecting procedure of the UWB radar with compressive sensing will be described and the singular value decomposition (SVD) of compressed UWB radar data will be presented for through-wall human detection in Section 3. Experimental results for human being detection under gypsum wall will be shown in Section 4. Conclusion and discussion is in Section 5.

\section{Compressive sensing theory}

Assuming signal $x \in R^{N}$, it can be expressed as:

$$
x=\sum_{i=1}^{N} \phi_{i} \theta_{i} \text { or } x=\Phi \theta
$$

where $\Phi$ is the $N \times N$ orthonormal transform basis and $\theta$ is the expansion coefficient vector in the orthonormal basis. If $K$ elements in vector $\theta$ are not zero and $K$ is far less than $N$, signal $x$ can be called a $K$ sparse signal.

Compressive sensing showed that the signal may be acquired with a small set of non-adaptive, linear measurements as long as the signal is sparse in some basis. So, we can capture the signal $x$ via linear measurements:

$$
y=\Psi x=\Psi \Phi \theta
$$

where $\Psi$ is a $M \times N$ random measurement matrix and $M<N$. Here, $(\Phi, \Psi)$ is a pair of orthobases which followed the incoherence restriction. The coherence
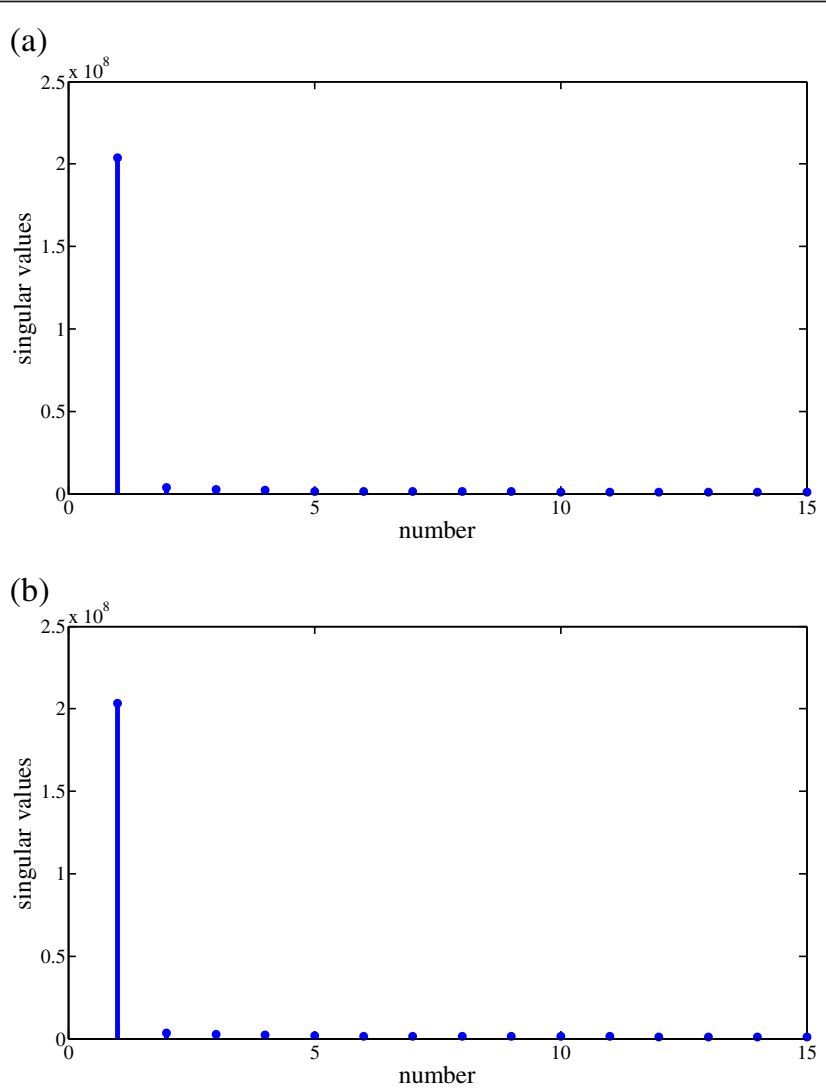

Figure 1 The singular values of compressed UWB radar data without target for gypsum wall. (a) The singular values of the first 15 compressed measurements. (b) The singular values of the second 15 compressed measurements. 
between the measurement basis $\Psi$ and the orthonormal transform basis $\Phi$ is [15-20]:

$$
\mu(\Psi, \Phi)=\sqrt{N} \cdot \max _{1 \leq k, j \leq N} \mid\left\langle\psi_{k}, \phi_{j}\right|
$$

The coherence measures the largest correlation between any two elements of $\Psi$ and $\Phi$. As for how large and how small, it follows from linear algebra that $\mu$ $(\Psi, \Phi) \in[1, \sqrt{N}]$. Also, compressive sensing is mainly concerned with low-coherence pairs.

If $x$ is termed as $K$ sparse in the orthonormal basis, we only need to collect $M=O(K \log (N / K))$ random measurements to recover the signal by searching for the $l_{0}$-sparsest that agrees with the measurements [21]:

$$
\hat{\theta}=\arg \min \|\theta\|_{0} \text { subject to } y=\Psi x=\Psi \Phi \theta
$$

where the $l_{0}$ pseudo-norm $\|\cdot\|_{0}$ corresponds to the number of non-zero elements. As we know, Equation 4 is a daunting NP-complete combinatorial optimization problem which is hardly solved directly in practice. Compressive sensing theory seeks to solve the closest possible' tractable minimization problem by solving the $l_{1}$-minimization, and it leads to a much simpler convex problem.

Compressive sensing theory shows that the signal can be sampled and compressed simultaneously. Also, the acquired low-dimensional signal contained the main features of the signal under the premise of an appropriate measurement matrix. Therefore, the CS theory provided an effective way to overcome the complexity of through-wall human detection in a large amount of UWB radar data.

\section{UWB radar data collected and SVD processing}

The through-wall detection of human being is based on the fact that the human body is always in a state of motion even if it sleeps or is trapped because of breathing. These tiny human motions would cause periodic changes of electromagnetic wave which is emitted by the UWB radar and returns through walls from the human body target [11]. Multiple echo signals of the UWB radar can reflect this periodic change. Thus, a $P \times N$
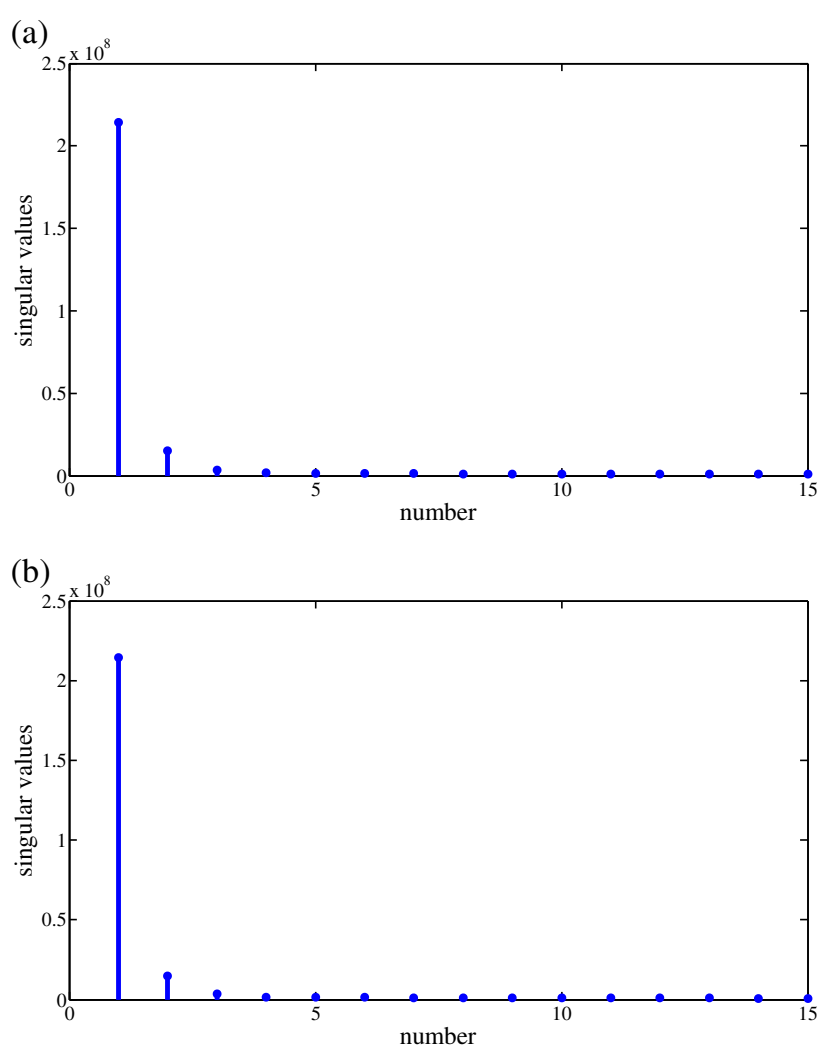

Figure 2 The singular values of compressed UWB radar data with target for gypsum wall. (a) The singular values of the first 15 compressed measurements. (b) The singular values of the second 15 compressed measurements. 

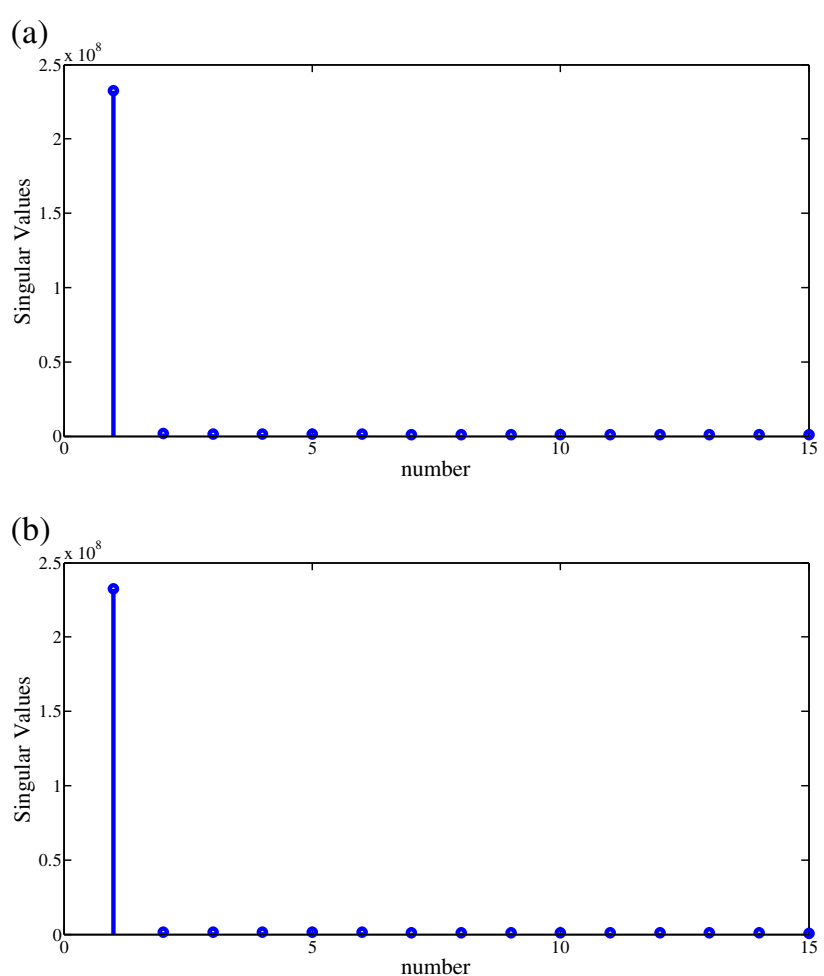

Figure 3 The singular values of compressed UWB radar data without target for brick wall. (a) The singular values of the first 15 compressed measurements. (b) The singular values of the second 15 compressed measurements.

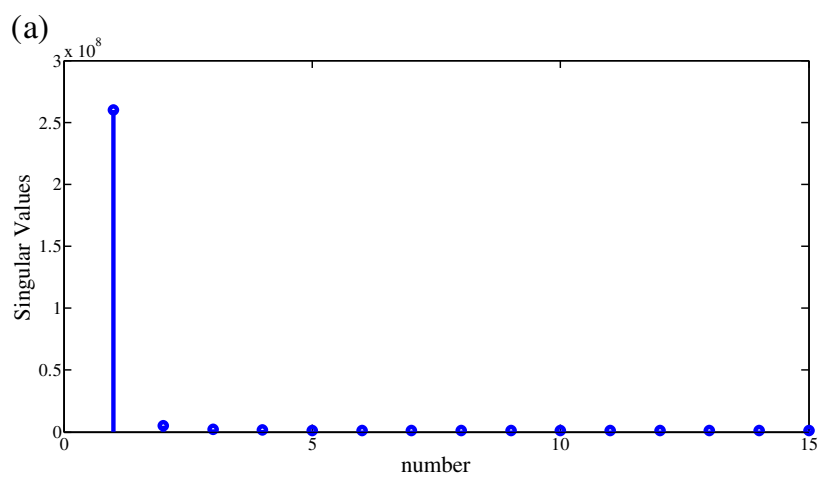

(b)

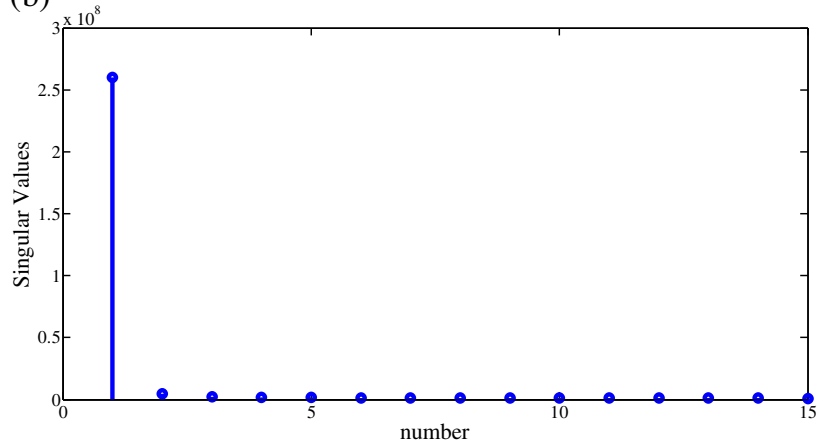

Figure 4 The singular values of compressed UWB radar data with target for brick wall. (a) The singular values of the first 15 compressed measurements. (b) The singular values of the second 15 compressed measurements. 

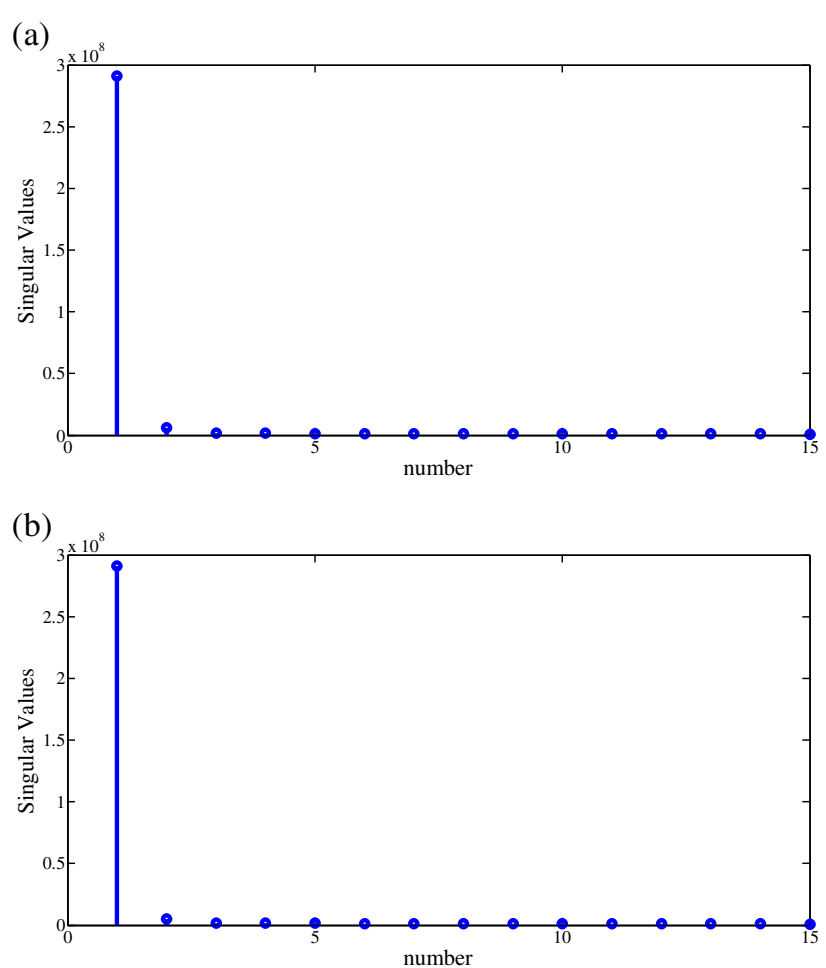

Figure 5 The singular values of compressed UWB radar data without target for concrete wall. (a) The singular values of the first 15 compressed measurements. (b) The singular values of the second 15 compressed measurements.
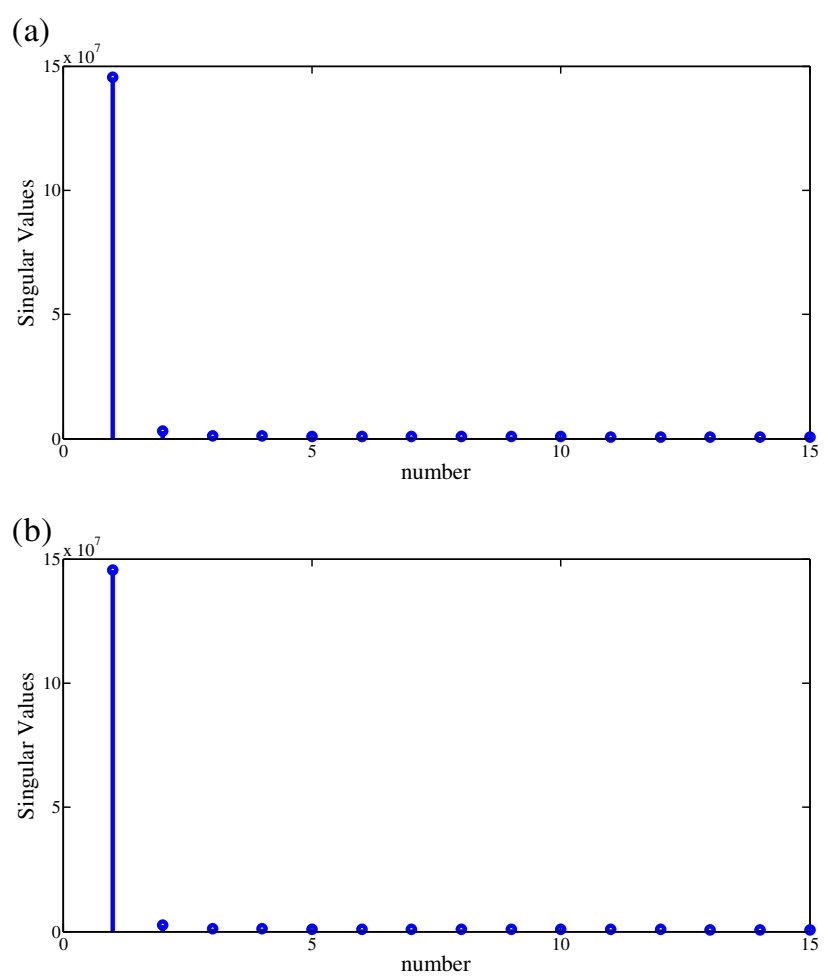

Figure 6 The singular values of compressed UWB radar data with target for concrete wall. (a) The singular values of the first 15 compressed measurements. (b) The singular values of the second 15 compressed measurements. 
matrix $E$ can be constructed using $P$ echoes with length $N$ of each echo:

$$
E=\left[\begin{array}{cccc}
s 11 & s 21 & \cdots & s P 1 \\
s 12 & s 22 & \cdots & s P 2 \\
\vdots & \vdots & \cdots & s P 3 \\
s 1 N & s 2 N & \cdots & s P N
\end{array}\right]
$$

With the random measurement matrix $\Psi \in Z^{N \times M}$, the compressed echo signals can be collected based on compressive sensing theory. Thus, a $P \times M$ matrix $D$ can be obtained.

SVD is an effective signal processing algorithm for the extraction of signal characteristics. The SVD of $D$ can be denoted as:

$$
D=U S V^{T}
$$

where the columns of $U$ are the eigenvectors of the $D D^{T}$ matrix and the columns of $V$ are the eigenvectors of the $D^{T} D$ matrix. $S$ is a diagonal matrix with the definition of the non-diagonal elements of diagonal matrices being zero. The diagonal elements of $S$ are a special kind of values of the original matrix. They are termed as the singular values of $D$.

Combining the reconstruction theory of CS and SVD theory, the singular values can indicate not only the signal characteristics of compressed matrix $D$ but also those of original matrix $E$. Therefore, through-wall human detection could be achieved by comparing the changes of singular values.

\section{Results and analysis}

In this paper, we used the P220 UWB radar (Time Domain Company, Huntsville, AL, USA) as the measurement tool which worked in monostatic mode in which waveform pulses were transmitted from a single omnidirectional antenna and the scattered waveforms were received by a collocated omnidirectional antenna. In the experiments, the P220 UWB radar worked in a center frequency of $4.3 \mathrm{GHz}$ with $10-\mathrm{dB}$ bandwidth at $2.3 \mathrm{GHz}$ and a resolution of $6.5 \mathrm{~cm}$.

In the project, we have taken measurements of through-wall detection for still human being with the P220 UWB radar at gypsum wall, brick wall, and concrete wall.

Each of the 30 measurements was taken with people and without a target. A random Gaussian matrix was used as the measurement matrix for compressed UWB radar data acquired. Then the SVD of the compressed data was implemented. The results are shown in Figures 1, 2, 3, 4,5 , and 6 .

Figures 1, 2, 3, 4, 5, and 6 show the singular values of 15 compressed measurements without a human target and with a human being where the compressive ratio is
0.5 . From the results, it can be seen that there were minor changes in singular values at the same status of 'with target' or 'without target' for the three kinds of walls. However, the singular value was increased at the status of 'with target' especially the main singular value. In other words, the singular values of compressed UWB radar data can be used for through-wall human detection for gypsum wall, brick wall, and concrete wall.

\section{Conclusion}

UWB radar is one of the most favorable methods for through-wall human detection due to its strong penetration ability. Because of the large amount of UWB radar data, the paper proposed the SVD algorithm for compressed UWB radar data to detect through-wall human being with compressive sensing theory. The experiments for human detection under the status for gypsum wall were tested, and the results demonstrated that the singular values could effectively reflect the presence of the human target.

\section{Competing interests}

The authors declare that they have no competing interests.

\section{Acknowledgements}

The authors would love to thank Professor Qilian Liang of the University of Texas at Arlington for providing the UWB radar data. This research was supported by the Tianjin Younger Natural Science Foundation (12JCQNJC00400) and National Natural Science Foundation of China (61271411).

Received: 20 May 2013 Accepted: 30 May 2013

Published: 12 June 2013

\section{References}

1. Q Liang, Biologically-inspired target recognition in radar sensor networks EURASIP Journal on Wireless Communications and Networking 2010, $523435(2010)$

2. Q Liang, X Cheng, S Samn, NEW: network-enabled electronic warfare for target recognition. IEEE Trans on Aerospace and Electronic Systems 46(2), 558-568 (2010)

3. Q Liang, Automatic target recognition using waveform diversity in radar sensor networks. Pattern Recognition Letters (Elsevier) 29(2), 377-381 (2008)

4. Q Liang, X Cheng, KUPS: knowledge-based ubiquitous and persistent sensor networks for threat assessment. IEEE Transactions on Aerospace and Electronic Systems 44(3), 1060-1069 (2008)

5. Q Liang, Waveform design and diversity in radar sensor networks: theoretical analysis and application to automatic target recognition, in IEEE Third Annual Conference on Sensor, Mesh and Ad Hoc Communications and Networks (SECON2006) (Reston, 25-28 Sept 2006)

6. E Zaikov, J Sachs, M Aftanas, J Rovnakova, Detection of trapped people by UWB radar, in German Microwave Conference (GeMIC), vol. 3 (Hamburg, 10-12 Mar 2008), pp. 1-4

7. J Rovnakova, M Svecova, D Kocur, $\Pi T$ Nguyen, J Sachs, Signal processing for through wall moving target tracking by M-sequence UWB radar, in 2008 18th International Conference on Radioelektronika, vol. 4 (Prague, Prague, 24-25 Apr 2008), pp. 1-4

8. D Kocur, J Gamec, M Svecova, M Gamcova, J Rovnakova, Imaging method: an efficient algorithm for moving target tracking by UWB radar. Acta Polytechnica Hungarica 7(3), 5-24 (2010)

9. D Kocur, J Rovñáková, M Svecová, Through wall tracking of moving targets by M-sequence UWB radar. Studies in Computational Intelligence $\mathbf{2 4 3}$ 349-364 (2009)

10. J Sachs, M Aftanas, S Crabbe, M Drutarovsky, R Klukas, D Kocur, TT Nguyen, P Peyer, J Rovnakova, E Zaikov, Detection and tracking of moving or 
trapped people hidden by obstacles using ultra-wideband pseudo-noise radar. European Radar Conference 10, 408-411 (2008)

11. S Singh, Q Liang, D Chen, L Sheng, Sense through wall human detection using UWB radar. EURASIP Journal on Wireless Communications and Networking 2011, 20 (2011)

12. W Wang, $X$ Zhou, B Zhang, J Mu, Anomaly detection in big data from UWB radars. Security and Communication Networks. in press

13. DL Donoho, Compressed sensing. IEEE Transactions on Information Theory 52(4), 1289-1306 (2006)

14. E Candès, Compressive sampling, in Proceedings of International Congress of Mathematicians (European Mathematical Society Publishing House, Madrid 2006), pp. 1433-1452

15. E Candès, M Wakin, An introduction to compressive sampling. IEEE Signal Processing Magazine 25, 21-30 (2008)

16. L Xu, Q Liang, X Cheng, D Chen, Compressive sensing in distributed radar sensor networks using pulse compression waveforms. EURASIP Journal of Wireless Communications and Networking 2013, 36 (2013). doi:10.1186/ 1687-1499-2013-36

17. L Xu, Q Liang, Zero correlation zone sequence pair sets for MIMO radar. IEEE Trans on Aerospace and Electronic Systems 48(3), 2100-2113 (2012)

18. L Xu, Q Liang, Orthogonal pulse compression codes for MIMO radar system, in IEEE Globecom (Miami, 6-10 Dec 2010)

19. L Xu, Q Liang, Waveform design and optimization in radar sensor network, in IEEE GLOBECOM (Miami, 6-10 Dec 2010)

20. Q Liang, JM Mendel, Design interval type-2 fuzzy logic systems using SVDQR method: rule reduction. International Journal of Intelligent Systems 15(10), 939-957 (2000)

21. A Averbuch, D Shai, D Shay, Adaptive compressed image sensing using dictionaries. SIAM Journal of Imaging Sciences 5(1), 57-89 (2012)

doi:10.1186/1687-1499-2013-162

Cite this article as: Zhang and Wang: Through-wall detection of human being with compressed UWB radar data. EURASIP Journal on Wireless Communications and Networking 2013 2013:162.

\section{Submit your manuscript to a SpringerOpen ${ }^{\circ}$ journal and benefit from:}

- Convenient online submission

- Rigorous peer review

- Immediate publication on acceptance

- Open access: articles freely available online

- High visibility within the field

- Retaining the copyright to your article

Submit your next manuscript at $\gg$ springeropen.com 